\title{
Évaluation de l'impact du rejet des déchets phosphates dans la mer sur la biodiversité marine dans trois localités côtières au Togo à partir des biomarqueurs du stress oxydatif chez Sphyraena barracuda (HECKEL, 1843)
}

\author{
Mamatchi MELILA $^{1^{*}}$, Wiyao POUTOULI ${ }^{3}$, Kou'santa Sabiba AMOUZOU ${ }^{1}$, \\ Tchangbédji GADO ${ }^{2}$, Macléwè TCHAO ${ }^{1}$ et Ananivi DOH ${ }^{1}$ \\ ${ }^{1}$ Laboratoire de biochimie appliquée à la nutrition et à l'alimentation, Département de Biochimie/Nutrition, \\ Faculté des Sciences, Université de Lomé, Togo. \\ ${ }^{2}$ Laboratoire de Gestion Traitement et Valorisation des Déchets (GTVD), Département de Chimie, \\ Faculté des Sciences, Université de Lomé, Togo. \\ ${ }^{3}$ Laboratoire de Biologie Animale, Faculté des Sciences, Université de Lomé, Togo. \\ *Auteur correspondant, E-mail: dominique2tg@yahoo.fr ; Tel: (228)90747178/(228)98273769.
}

\section{RESUME}

La pollution due aux rejets des déchets phosphatés à Kpémé par la Société Nouvelle des Phosphates du Togo (SNPT), concerne aussi les zones environnantes. Nous avons voulu dans ce travail déterminer l'impact de cette pollution dans ces zones sur la biodiversité marine. Les teneurs en métaux lourds toxiques $(\mathrm{Cd}$ et $\mathrm{Pb})$ dans les organes de poissons à Gbodjomé (zone de référence), Agbodrafo, Goumoukopé et Aného sont déterminées par spectrophotométrie d'absorption atomique à la flamme; de même que certains biomarqueurs du stress oxydatif. Dans l'ensemble, les résultats ont montré une altération des enzymes antioxydantes, du système de la glutathion et une induction de la peroxydation lipidique due à la présence du $\mathrm{Cd}$ et $\mathrm{du} \mathrm{Pb}$ qui provoquent un stress oxydatif chez les poissons, et donc chez les espèces de la biodiversité marine de la côte togolaise des zones concernées. Les résultats de la présente étude indiquent que les zones environnantes sont touchées par le rejet des déchets à Kpémé et les impacts sur la biodiversité marine diminuent au fur et à mesure qu'on s'éloigne des sites de rejets vers l'aval à cause du sens d'écoulement de la mer. Aussi, peut-on déduire que les biomarqueurs du stress oxydatif sont des indicateurs de la pollution aquatique.

(C) 2012 International Formulae Group. All rights reserved.

Mots clés : Déchets phosphatés, pollution, $\mathrm{Cd}, \mathrm{Pb}$, bioaccumulation.

\section{INTRODUCTION}

La pollution aquatique est un facteur majeur du stress oxydatif de la faune aquatique et en particulier chez les poissons (Yildirim et al., 2011). En effet, lorsque l'organisme fonctionne bien, il y a un équilibre constant entre la production des ROS «reactive oxygen species» due aux xénobiotiques et les mécanismes de leur élimination (système oxydant) (Ahmed et al., 2000). Cet équilibre, en cas d'une pollution environnementale, peut être rompu en faveur du système pro-oxydant, instaurant ainsi un état de stress oxydatif (Ait-Aïssa et al., 2003). Ainsi, pour neutraliser les effets toxiques des radicaux libres, les poissons comme les 
mammifères possèdent un système d'antioxydants (Nordberg et Arner, 2001; Almeida et al., 2002 ; Pandey et al., 2003) qui comprend des enzymes antioxydantes comme le superoxyde dismutase (SOD); la catalase (CAT) ; la glutathion peroxydase (GSH-Px) ; la glutathion-S-transférase (GST) et la glutathion réductase (GR). En outre, de nombreuses molécules comme la glutathion (GSH), les vitamines A, C et $\mathrm{E}$ peuvent participer au processus d'élimination des oxyradicaux (Yildirim et al., 2011). Les systèmes cellulaires de défenses antioxydantes dans les organismes biologiques sont altérés lorsqu'ils sont exposés aux polluants environnementaux ; mais les niveaux d'antioxydants dans les organismes vivants peuvent augmenter de manière à restaurer le déséquilibre causé par les dommages oxydatifs (Yildirim et al., 2011). Les teneurs en enzymes antioxydantes peuvent donc être utilisées comme biomarqueurs du stress oxydatif (Livingstone, 2001). Lorsque les défenses antioxydantes sont affaiblies ou dépassées, le stress oxydatif peut provoquer l'inactivation enzymatique et la péroxydation lipidique (Halliwell et Gutteridge, 1989). Par ailleurs, la Malondialdéhyde (MDA), utilisée comme marqueur de l'oxydation des phospholipides membranaires à partir de la peroxydation lipidique, reflète le statut oxydatif des espèces exposées aux xénobiotiques (Sole et al., 1996 ; Charissou et al., 2004). Le niveau des enzymes antioxydantes a été largement utilisé comme un indicateur d'alerte précoce de la pollution aquatique (Lin et al., 2001), et les poissons ont été proposés comme indicateurs de suivi de la pollution aquatique car ils peuvent concentrer des polluants dans leur tissus directement à partir de l'eau par la respiration, mais aussi à travers leur alimentation (Farombi et al., 2007 ; Velkova-Jordanoska et al., 2008 ; Yildirim et al., 2011). Il a été démontré d'autre part que les activités de la SOD, de la CAT, de même que la concentration de la glutathion et la formation de la MDA dans les organes de poissons sont des indicateurs de la pollution de l'environnement aquatique
(Farombi et al., 2007). Aussi, Yildirim et al. (2011) ont montré que l'altération des enzymes antioxydantes, de la glutathion et l'induction de la peroxydation lipidique reflètent la présence des métaux lourds qui peuvent provoquer le stress oxydatif chez les espèces aquatiques. Au Togo, des études ont montré que les boues phosphatées rejetées à Kpémé après le traitement du minerai brut de phosphate par l'usine de la SNPT et contenant les métaux lourds toxiques (Plomb $\ll \mathrm{Pb} »$ et Cadmium «Cd») peuvent induire chez les espèces marines à ce niveau des conséquences biochimiques (Abbe, 2004) tels qu'un stress oxydatif. Or, ces boues sont drainées par les eaux de part et d'autre de la zone de rejet jusqu'au niveau des villages limitrophes de Kpémé, Agbodrafo vers l'ouest, et Goumoukopé à l'est et même au-delà jusqu'à Aného. Afin d'avoir une idée sur le niveau de la pollution marine au niveau de ces zones concernées par les rejets dans la mer des déchets issus du traitement du minerai de phosphate, nous avons évalué dans ce travail les niveaux d'impact sur la faune aquatique des deux villages limitrophes et au niveau d'Aného. Pour se faire, nous nous sommes référés au stress oxydatif à partir de la réponse de l'activité des enzymes antioxydantes et la peroxydation lipidique chez une espèce de poisson ( $S$. barracuda). Dans la présente étude, nous avons déterminé l'activité de la GST, l'activité de la CAT, la concentration de la GSH et la formation de la MDA dans le foie, les branchies, les reins et le cœur de $S$. barracuda des eaux marines de la côte togolaise à Agbodrafo, à Goumoukopé et à Aného (zones concernées par les rejets de déchets phosphatés), de même qu'à Gbodjomé, zone de référence située à environ $11 \mathrm{Km}$ à l'ouest et en amont de l'usine de la SNPT.

\section{MATERIEL ET METHODES}

\section{Le cadre de l'étude}

La zone d'étude est un ensemble situé du côté Sud de la route nationale LoméAného, allant du village de Gbodjomé (Ouest) jusqu'à Aného (Est) en passant par Kpémé sur 
une distance d'environ $20 \mathrm{Km}$. Par sa situation géographique, cet ensemble fait partie de la préfecture des Lacs dans la région Maritime du Togo (Figure 1). La caractéristique essentielle de cet espace géographique est qu'il abrite l'usine de traitement du minerai de phosphate de Hahotoé (Togo) dont les déchets et les boues, rejetés dans la mer (Figure 2), contiennent le $\mathrm{Pb}$ et le $\mathrm{Cd}$, métaux lourds très toxiques.

\section{Matériel}

Il s'agit des échantillons d'eau de mer prélevés à Agbodrafo, Goumoukopé et Aného, zones concernées par les rejets des déchets phosphatés, et à Gbodjomé zone servant de contrôle, située en amont à environs $11 \mathrm{Km}$ de l'usine de la SNPT et qui ne fait donc pas objet de rejet des déchets phosphatés; des échantillons de poissons ( $S$. barracuda), achetés vivants chez les pêcheurs de la côte togolaise au niveau de ces localités. Cette espèce de poisson, localement appelée «Lizi », a été identifiée au Département de Biologie Animale de l'Université de Lomé;

- Des appareils et matériel de laboratoire ;

- Des réactifs de laboratoire tels que : le $\beta$-2-mercaptoétanol, le TritonX100, le peroxyde d'hydrogène, le tampon Tris- $\mathrm{HCl}$, l'acide chlorhydrique, la solution d'Ethyle Diamine Tétra-Acétique (EDTA), l'acide nitrique, le chloro-2,4-dinitrobenzene (CDNB) qui sont fournis par LABOSI OULSHY-LECHATEAU-France; l'éthanol absolu, le réactif de Bradford, le Sérum Albumine Bovine (BSA) qui sont fournis par Biolabo S.A. FISMES (France); l'acide 5,5'-dithiobis-2-nitrobenzoïque, le dodécyle sulfate sodium (SDS), la malonedialdéhyde (MDA) qui sont fournis par SIGMA Chemical (St louis, USA) ; les solutions étalons de plomb et de cadmium proviennent de Merck KGaA (Durmstalt, Allemagne).

\section{Echantillonnage}

Les poissons adultes vivants ont été sélectionnés et achetés chez les pêcheurs de la côte togolaise au niveau de Gbodjomé,
Agbodrafo, Goumoukopé et Aného (10 poisons par zone), puis transportés le même jour au laboratoire dans des bacs à la température ambiante; ils y sont gardés vivants au moins 24 heures pour minimiser le stress. Ces poissons sont ensuite disséqués et le foie, le cœur, les branchies et les reins sont rapidement prélevés et regroupés par lots. Chaque lot d'organes est réparti en deux fractions: une première est réservée à la minéralisation pour la détermination des teneurs en $\mathrm{Cd}$ et $\mathrm{Pb}$ et une deuxième fraction est traitée pour les dosages protéiques et des bioindicateurs du stress oxydatif (enzymatiques et non enzymatiques). Les prélèvements d'eau de mer sont réalisés dans chaque localité dans les flacons de $50 \mathrm{ml}$ contenant $2 \mathrm{ml}$ d'acide nitrique $\left(\mathrm{HNO}_{3}\right)$ pour éviter une éventuelle précipitation et/ou complexassion des métaux lourds pendant la période de conservation.

\section{Méthodologie \\ Minéralisation des échantillons et détermination des teneurs en $\mathrm{Cd}$ et en $\mathrm{Pb}$.}

Chaque fraction de chaque lot d'organes réservés à cet effet est placée à l'étuve à $50{ }^{\circ} \mathrm{C}$ pendant 96 heures puis pesée pour déterminer la masse sèche avant d'être réduite en poudre par broyage dans un mortier. $1 \mathrm{~g}$ de la poudre obtenue est additionné à $5 \mathrm{ml}$ de $\mathrm{HNO}_{3}(18 \mathrm{M})$ dans des téflons recouverts de verres de montre placés sur un bain de sable, dispositif chauffant pendant environ trois heures pour la digestion du tissu. Le produit de la digestion est récupéré avec quelques $\mathrm{ml}$ d'eau distillée, filtré et repris dans un volume final de $50 \mathrm{ml}$ en ajoutant de l'eau distillée. La solution ainsi obtenue est utilisée pour la lecture au spectrophotomètre d'absorption atomique à la flamme (modèle AA Sol Aar; Thermo Electron Corporation ; Allemagne) couplé à un micro-ordinateur.

\section{Dosage protéique et des bioindicateurs du stress oxydatif \\ Chaque fraction de chaque lot d'organes réservée a cet effet est lavée dans une solution tampon (Tris- $\mathrm{HCl} 10 \mathrm{Mm}$. PH}


7 ,5) supplémentée en EDTA $(3,8$ g /1), $\beta-2-$ mercaptoétanol $(348 \mu \mathrm{l} / \mathrm{l})$ et en Triton X100 (1 $\mathrm{ml} / \mathrm{l})$ puis broyée au poter. L'homogénat tissulaire ainsi obtenu est additionné de $1 \mathrm{ml}$ du même tampon et centrifugé à $3000 \mathrm{trs} / \mathrm{min}$ pendant 10 minutes. Après cette centrifugation, le surnageant et le culot sont séparés et les deux fractions sont conservées à $-20{ }^{\circ} \mathrm{C}$ jusqu'à leur utilisation. Le surnageant a servi au dosage des protéines, des enzymes et de la glutathion (GSH) alors que la malonedialdéhyde (MDA) a été dosée dans le culot.

La teneur en protéines totales des fractions a été estimée par la méthode de Bradford (1976) avec le sérum albumine bovine (BSA) comme standard.

La Glutathion $(\mathrm{GSH})$ a été déterminée dans la fraction de foie, reins, branchies et cœur homogénéisée de $S$. barracuda selon la méthode de Jollow et al. (1974) à $412 \mathrm{~nm}$ en utilisant l'acide 5,5'-dithio-bis-2nitrobenzoïque (DTNB).

Pour déterminer l'activité de la glutathion-s-transférase (GST), nous avons utilisé la méthode de Habig et al. (1974) avec le 1-chloro-2,4-dinitrobenzene (CDNB) comme substrat.

L'activité de la catalase est déterminée par la méthode d'Aebi (1984) basée sur la propriété de l'enzyme à dégrader le peroxyde d'hydrogène $\left(\mathrm{H}_{2} \mathrm{O}_{2}\right)$ qui est son substrat naturel. La disparition du substrat est alors suivie par spectrophotométrie à $240 \mathrm{~nm}$ au moyen d'un lecteur de plaques (modèle Spectra Max molecular Device, Sunyval Corporation, California USA).

La peroxydation des lipides (MDA) a été déterminée en mesurant les substances acides réagissant dans l'acide thiobarbiturique (TBARS) comme décrit par Satoh (1978).

\section{Analyse statistique}

Tous les résultats sont exprimés sous forme de la moyenne \pm ESM et analysés à l'aide du logiciel Graph pad Prism. L'analyse de la variance a été effectuée à l'aide du Test t de Student unpaired. Le seuil de signification est fixé à $\mathrm{P}<0,05$.

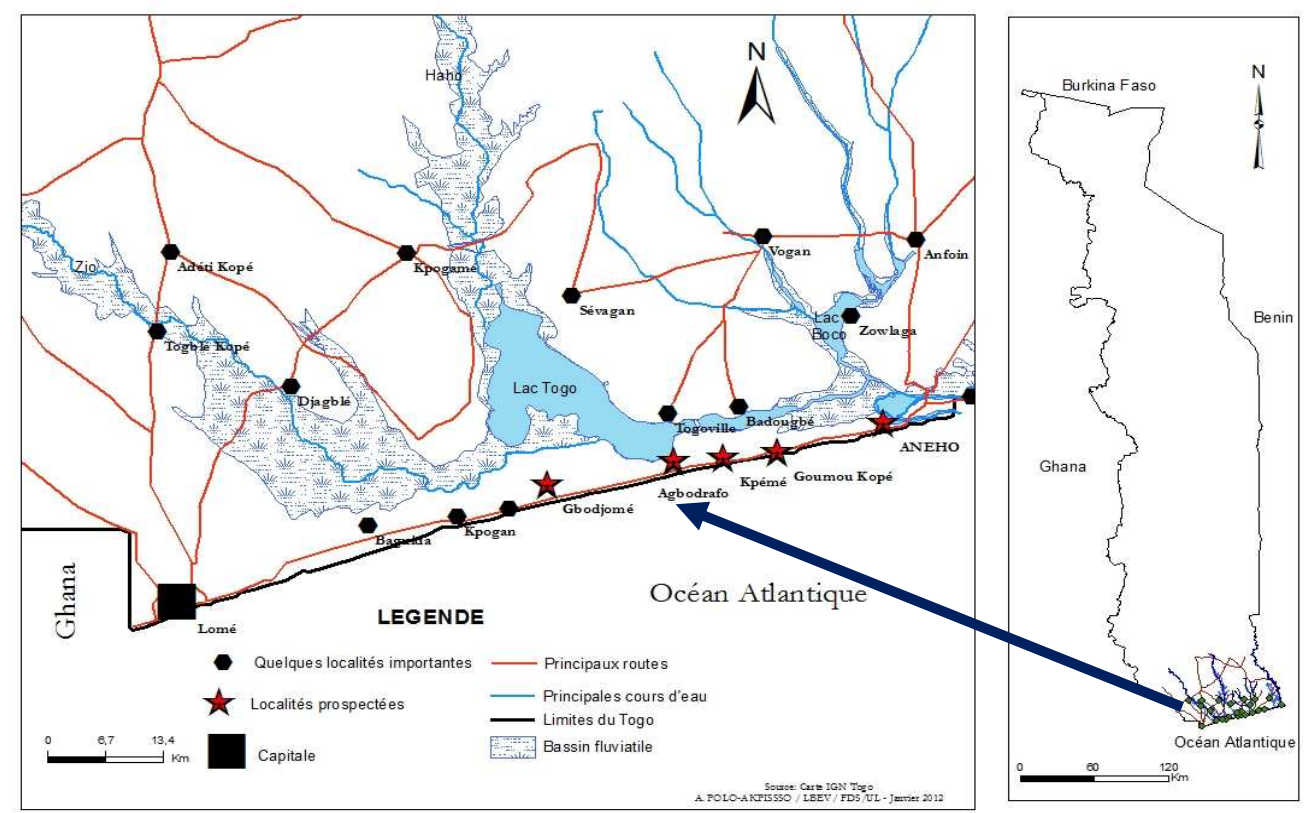

Figure 1 : Carte indiquant les zones d'étude concernées par le rejet des déchets phosphatés. 


\section{RESULTATS}

Teneurs en $\mathrm{Cd}$ et en $\mathrm{Pb}$ de l'eau de mer et des différents organes des poissons

Les teneurs en $\mathrm{Cd}$ et en $\mathrm{Pb}$ dans l'eau de mer à Gbodjomé, Agbodrafo, Goumoukopé et Aného sont indiquées dans le Tableau 1 et celles des organes de poissons (foie, reins, cœur et branchies) des mêmes localités sont indiquées dans le Tableau 2. Les teneurs en $\mathrm{Cd}$ et en $\mathrm{Pb}$ de l'eau de mer à Agbodrafo, Goumoukopé et Aného (zones de pollution) sont plus élevées que celles de Gbodjomé et plus élevées que les normes admises (WHO, 1992). Dans les différents organes de poissons, les teneurs de $\mathrm{Pb}$ sont supérieures à celles du Cd (Tableau 2). L'ordre de concentration des métaux dans les organes se présente comme suit : $-\mathrm{Cd}$ : foie $>$ branchies $>$ reins $>$ cœur ; $-\mathrm{Pb}$ : foie $>$ reins $>$ branchies $>$ cœur.

Les concentrations du $\mathrm{Cd}$ et du $\mathrm{Pb}$ dans tous les organes (Tableau 2) sont plus élevées que les normes autorisées pour la consommation (WHO, 1992) au niveau des zones de pollution.

\section{Taux de la glutathion (GSH)}

Les taux de la GSH dans le foie, les reins, les branchies et le cour de $S$. barracuda sont présentés dans la Figure 3.

Les résultats montrent que :

- A Agbodrafo, les taux de la GSH ont augmenté d'une façon significative dans le foie de 58\% ( $\mathrm{P}<0,001)$, dans les reins de $74 \%(\mathrm{P}<0,001)$ et dans le cœur de 30\% ( $\mathrm{P}<$ $0,01)$ mais ont diminué significativement $(\mathrm{P}<$ $0,05)$ dans les branchies de $15 \%$ par rapport aux taux obtenus dans les mêmes organes à Gbodjomé (Figure 3).

- A Goumoukopé, on note aussi une augmentation des taux de la GSH significativement dans le foie de $71 \%$ ( $\mathrm{P}<$ $0,001)$, dans les reins de $93 \%(\mathrm{P}<0,001)$ et dans le cœur de $41 \%(\mathrm{P}<0,001)$ et une diminution de ce taux dans les branchies de $19 \%(\mathrm{P}<0,05)$.

- A Aného on note une augmentation du taux de la GSH dans tous les 4 organes qui est de $32 \%(\mathrm{P}<0,01)$ dans le foie, de $45 \%$ ( $\mathrm{P}$ $<0,001)$ dans les reins, de $21 \%(\mathrm{P}<0,05)$ dans le cœur et de $22 \%(\mathrm{P}<0,01)$ dans les branchies.

\section{Taux de la Malonedialdéhyde (MDA)}

Les taux de la MDA obtenus dans les différents organes sont présentés dans la Figure 4.

Le taux de MDA a significativement augmenté dans tous les organes de $S$. barracuda avec une augmentation plus significative dans les organes de poissons de goumoukopé $(\mathrm{P}<0,001)$ par rapport à Gbodjomé. Les pourcentages d'augmentation de la peroxydation des lipides comparés à ceux de Gbodjomè dans le foie, les reins, le cœur et les branchies sont respectivement de $60 \% ; 64 \% ; 19 \%$ et $52 \%$ à Agbodrafo, de $187 \% ; 128 \%$; $56 \%$ et $154 \%$ à Goumoukopé et de $70 \% ; 76 \% ; 19 \%$ et $46 \%$ à Aného (Figure 4).

Activités des enzymes antioxydantes : la glutathion-s-transférase (GST) et la catalase (CAT)

Ces activités sont représentées respectivement par les Figures 5 et 6 . Les taux de la GST dans le foie, les reins et le cour de $S$. barracuda ont augmenté respectivement de $27 \%, 28 \%$ et $09 \%$ à Agbodrafo, de $43 \%$; $48 \%$ et $18 \%$ à Goumoukopé par rapport à ceux obtenus à Gbodjomé. Cependant, on note plutôt une baisse de $12 \%$ dans les branchies des poissons des deux sites $(\mathrm{P}<0,05)$. A Aného, on note plutôt une augmentation des taux de la GST de $14 \%, 23 \%, 3 \%$ et de $23 \%$ respectivement dans le foie, les reins, le cœur et les branchies par rapport à ceux obtenus à Gbodjomé.

Les taux de la catalase obtenus dans les organes de $S$. barracuda à Goumoukopé sont plus faibles que ceux obtenus à Gbodjomé. La réduction est respectivement de $27 \%, 16 \%$, $14 \%$ dans le foie, les reins, les branchies et de $37 \%$ dans le cœur. Par contre, ces taux ont plutôt augmentés par rapport à ceux de Gbodjomé au niveau d'Agbodrafo et Aného. L'augmentation est respectivement dans le foie, les reins, le cœur et les branchies de $35 \%, 22 \%, 11 \%$ et $78 \%$ à Agbodrafo, de $38 \%$, $21 \%, 10 \%$ et $54 \%$ à Aného. 


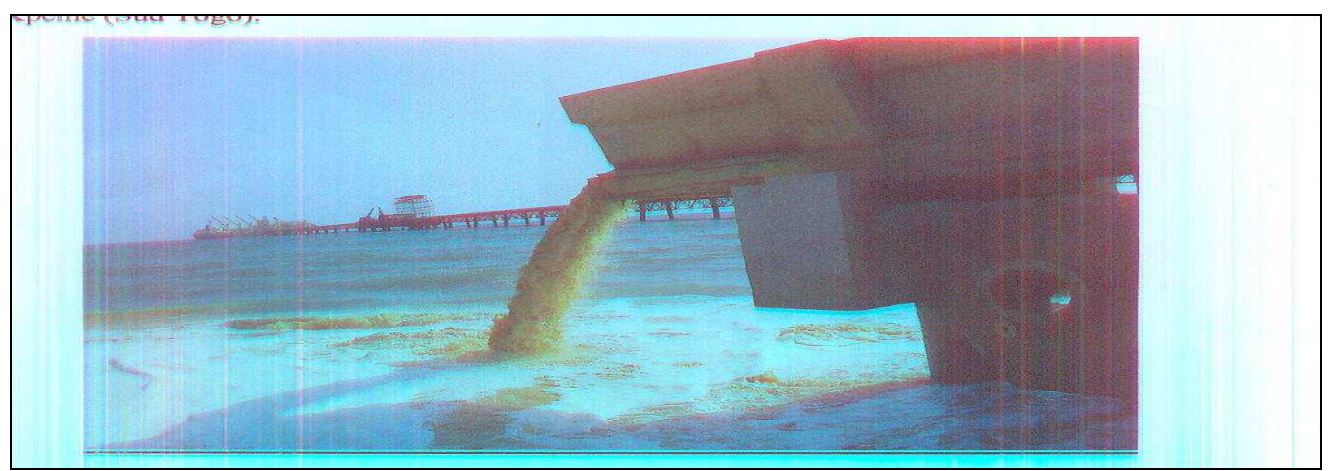

Figure 2 : Photographie montrant le rejet des boues phosphatées dans la mer à Kpémé.

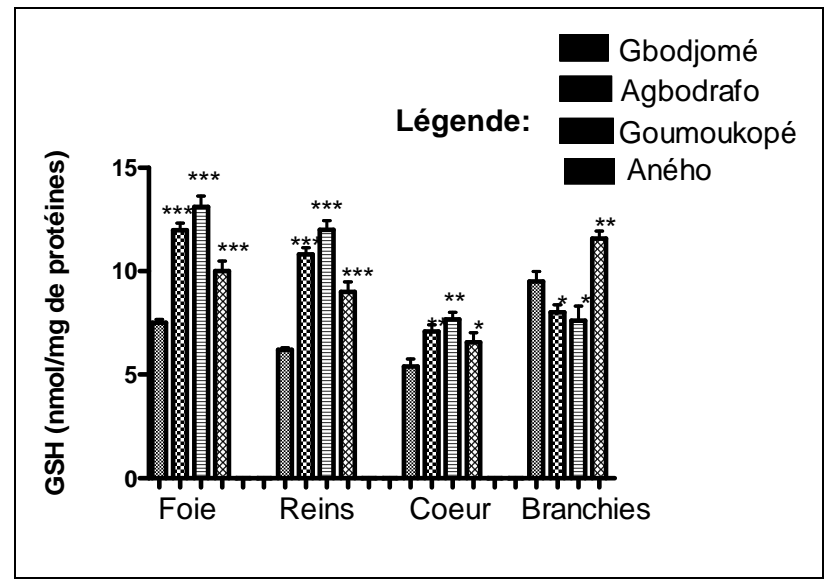

Figure 3 : Taux de la glutathion (GSH) dans les organes de Sphyraena barracuda selon les zones. Les valeurs sont exprimées sous forme de moyenne \pm ESM de 10 poissons. Significativement différent par rapport au contrôle : *** $\mathrm{P}<0,001 ; * * \mathrm{P}<0,01$ et $* \mathrm{P}<0,05$.

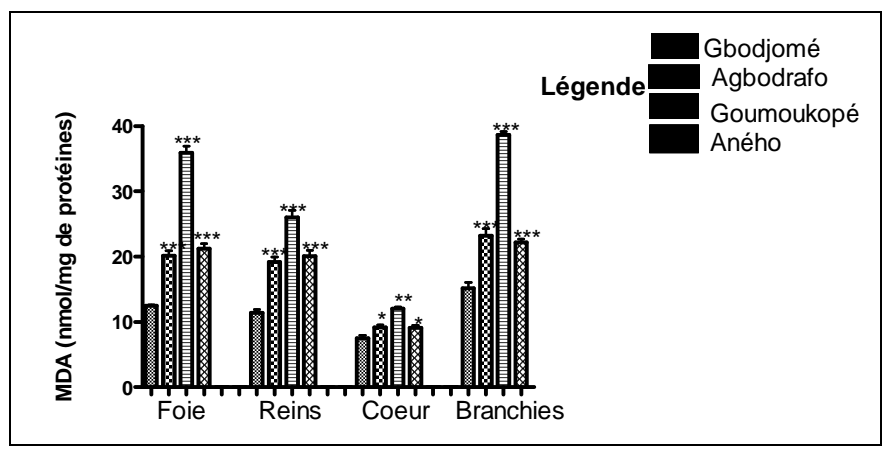

Figure 4 : Taux de la malonedialdéhyde (MDA) dans les organes de Sphyraena barracuda selon les zones. Les valeurs sont exprimées sous forme de moyenne \pm ESM de 10 poissons. Significativement différent par rapport au contrôle : *** $\mathrm{P}<0,001 ; * * \mathrm{P}<0,01$ et $* \mathrm{P}<0,05$. 


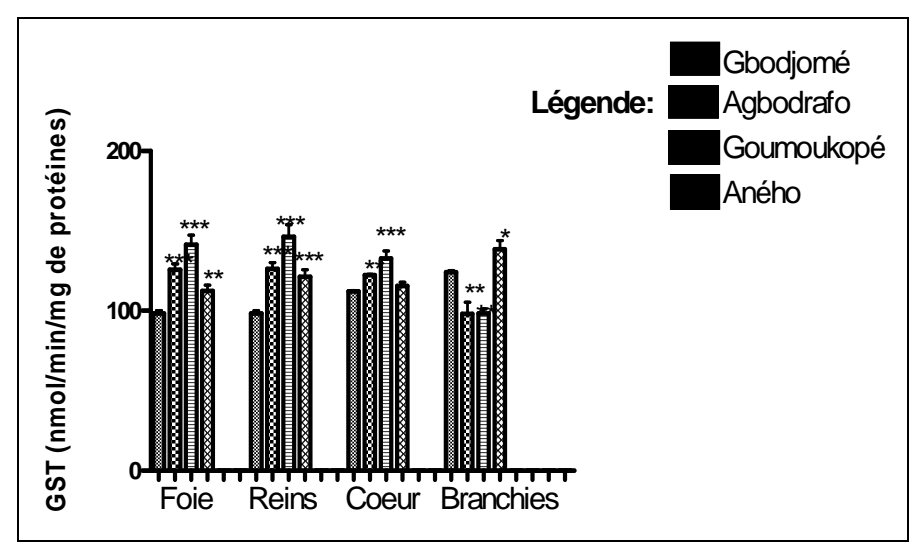

Figure 5 : Taux de la glutathion-S-transférase (GST) dans les organes de Sphyraena barracuda selon les zones. Les valeurs sont exprimées sous forme de moyenne \pm ESM de 10 poissons. Significativement différent par rapport au contrôle : *** $\mathrm{P}<0,001 ; * * \mathrm{P}<0,01$ et $* \mathrm{P}<0,05$.

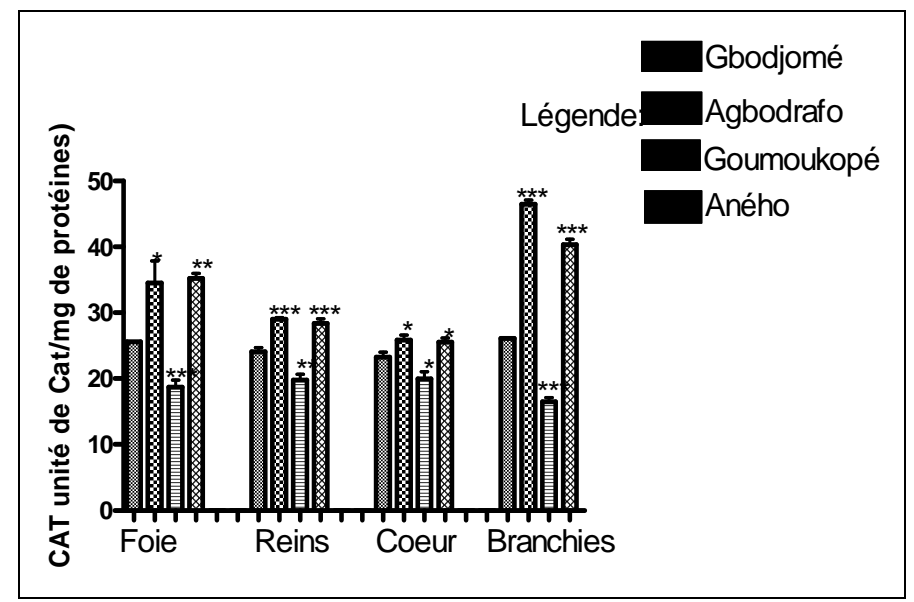

Significativement différent par rapport au contrôle : *** $\mathrm{P}<0,001 ; * * \mathrm{P}<0,01$ et $* \mathrm{P}<0,05$.

Figure 6 : Taux de la catalase (CAT) dans les organes de Sphyraena barracuda selon les zones. Les valeurs sont exprimées sous forme de moyenne \pm ESM de 10 poissons.

Tableau 1: Teneurs en $\mathrm{Cd}$ et en $\mathrm{Pb}$ de l'eau de mer à Gbodjomé ; Agbodrafo ; Goumoukopé et Aného.

\begin{tabular}{lccccc}
\hline $\begin{array}{l}\text { Teneurs en } \\
\text { métaux }(\boldsymbol{\mu g} / \mathbf{l})\end{array}$ & Gbodjomé & Agbodrafo & Goumoukopé & Aného & Normes \\
\hline $\mathrm{Pb}$ & $0,33 \pm 0,02$ & $6,09 \pm 0,930 * * *$ & $6,59 \pm 0,475^{* * *}$ & $4,03 \pm 0,367 * * *$ & 0,5 \\
$\mathrm{Cd}$ & $\mathrm{ND}$ & $0,83 \pm 1,030$ & $0,97 \pm 0,147$ & $0,57 \pm 0,120$ & 0,2 \\
\hline
\end{tabular}

Les résultats sont exprimés sous forme de moyenne \pm ESM de 10 essais. Significativement différent : *** $\mathrm{P}<0,001 . \mathrm{ND}=$ Non Déterminé (teneur inférieure au seuil de détection de l'appareil). 
Tableau 2: Les niveaux des teneurs en $\mathrm{Cd}$ et en $\mathrm{Pb}$ dans les organes de $S$. barracuda.

\begin{tabular}{|c|c|c|c|c|}
\hline \multirow{2}{*}{$\begin{array}{l}\text { Métaux et } \\
\text { localités }\end{array}$} & \multicolumn{4}{|c|}{ Organes de poissons } \\
\hline & Foie & Reins & Cœeur & Branchies \\
\hline \multicolumn{5}{|l|}{$\mathrm{Pb}(\mu \mathrm{g} / \mathrm{g})$} \\
\hline Gbodjomé & $0,03 \pm 0,008$ & ND & ND & $0,01 \pm 0,050$ \\
\hline Agbodrafo & $6,97 \pm 1,012 * * *$ & $6,01 \pm 0,904$ & $3,43 \pm 1,201$ & $4,55 \pm 0,874 * * *$ \\
\hline$\%$ de différence & 23133 & - & - & 45400 \\
\hline Goumoukopé & $7,48 \pm 0,991 * * *$ & $6,30 \pm 1,123$ & $3,67 \pm 0,365$ & $4,95 \pm 0,871$ *** \\
\hline$\%$ de différence & 24833 & - & - & 49400 \\
\hline Aného & $4,09 \pm 1,042 * * *$ & $3,57 \pm 0,298$ & $1,98 \pm 0,197$ & $3,01 \pm 0,569 * * *$ \\
\hline$\%$ de différence & 13533 & - & - & 30000 \\
\hline \multicolumn{5}{|l|}{$\mathrm{Cd}(\mu \mathrm{g} / \mathrm{g})$} \\
\hline Gbodjomé & $0,01 \pm 0,050$ & ND & ND & $0,01 \pm 0,050$ \\
\hline Agbodrafo & $0,81 \pm 0,773^{* * *}$ & $0,79 \pm 0,080$ & $0,07 \pm 0,005$ & $0,97 \pm 1,010^{* * *}$ \\
\hline$\%$ de différence & 8000 & - & - & 9600 \\
\hline Goumoukopé & $0,98 \pm 0,094 * * *$ & $1,01 \pm 0,920$ & $0,08 \pm 0,095$ & $1,09 \pm 0,517 * * *$ \\
\hline$\%$ de différence & 9700 & - & - & 10800 \\
\hline Aného & $0,63 \pm 0,068^{* * *}$ & $0,57 \pm 0,091$ & $0,06 \pm 0,007$ & $0,65 \pm 0,009 * * *$ \\
\hline$\%$ de différence & 6200 & 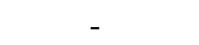 & - & 6400 \\
\hline
\end{tabular}

Les résultats sont exprimés sous forme de moyenne \pm ESM de 10 essais. Significativement différent : *** $\mathrm{P}<0,001$. $\mathrm{ND}=$ Non Déterminé (teneur inférieure au seuil de détection de l'appareil).

\section{DISCUSSION}

Les effluents industriels sont parmi tant d'autres les sources d'entrée des métaux lourds toxiques tels que le $\mathrm{Cd}$, le $\mathrm{Pb}$ (Chinni et al., 2000). Au Togo, la côte marine est polluée du fait, entre autres des activités industrielles, et des études sont menées concernant cette pollution notamment celle due aux activités industrielles de la SNPT qui traite le phosphate togolais (Gnandi, 1998 ; Tchangbédji et al., 2003; Abbe, 2004 ; Awadé, 2010). Cependant, l'évaluation des différentes concentrations des éléments traces métalliques tels que le $\mathrm{Cd}$ et le $\mathrm{Pb}$ que contiennent les boues phosphatées dans les différents organes physiologiques des espèces marines n'a pas encore été faite et il n'existe aucune donnée sur les biomarqueurs du stress oxydatif, bioindicateurs de la pollution aquatique (Farombi et al., 2007) et conséquence de la bioaccumulation des métaux lourds toxiques (Yildirim et al., 2011) dans les zones faisant l'objet de cette pollution. Dans cette étude, nous avons évalué les teneurs du $\mathrm{Cd}$ et du $\mathrm{Pb}$, principaux métaux lourds toxiques contenus dans les boues et déchets du phosphate togolais (Gnandi, 1998) et bioaccumulés par la biodiversité marine (Abbe, 2004), de même que le taux de certains biomarqueurs du stress oxydatif dans le foie, les reins, les branchies et le cour de $S$. barracuda, l'une des espèces de poisson de la côte marine togolaise très appréciées par les consommateurs et donc d'une importance commerciale non négligeable.

Nos résultats indiquent que le $\mathrm{Cd}$ et le $\mathrm{Pb}$ ont des niveaux de concentrations élevés dans tous les organes considérés de $S$. barracuda dans les zones touchées par cette pollution (Agbodrafo, Goumoukopé et Aného), par rapport à Gbodjomé, zone de contrôle; ce qui a induit une peroxydation lipidique de même qu'une altération des enzymes antioxydantes dans les organes des poissons. 
La concentration élevée des métaux dans les branchies peut s'expliquer par le fait que les branchies servent d'organes respiratoires chez les poissons, organes à travers lesquels les ions métalliques sont absorbés (Bebianno et al., 2004 ; Farombi et al., 2007). Les branchies étant donc en contact direct avec l'eau de mer et ayant un épithélium fin; ainsi les métaux peuvent facilement pénétrer à travers les cellules de ces dernières (Bebianno et al., 2004). La teneur élevée des métaux dans le foie serait due à une bioaccumulation car le foie est le centre de métabolisme et donc peut concentrer les métaux lourds (Farombi et al., 2007). La teneur élevée des métaux dans le foie et les branchies dans nos résultats, confirme les résultats des travaux antérieurs sur le poisson chat au Nigéria (Farombi et al., 2007). Aussi, la forte concentration des métaux dans le foie est signalée par des travaux sur sept espèces de poissons des eaux costales de l'Uruguay (Viana, 2005). Les teneurs élevées du Cd et $\mathrm{du} \mathrm{Pb}$ dans le foie et les reins de $S$. barracuda montrent que le foie et les reins possèdent une capacité d'accumulation des métaux à cause des protéines qui facilitent l'agglutination de ceux-ci (Kargin, 1999). Nos résultats dans ces deux organes de poissons confirment ceux de certaines études antérieures (Farombi et al., 2007). Les concentrations du Cd des organes au niveau des zones Agbodrafo, Goumoukopé, Aného sont non négligeables et se situent dans l'intervalle rapporté dans les études antérieures (Dautremepuits et al., 2004 ; Asharaf, 2005 ; Farombi et al., 2007). Dans une autre étude similaire, Kalay et al. (1999) ont rapporté une teneur du Cd de 1,98 ppm dans le foie du Mullus barbatus de la région côtière de la méditerranée alors que Farombi et al. (2007) ont trouvé des teneurs de 2,10 ppm, 0,69 ppm, 2,05 ppm et 0,25 ppm respectivement dans le foie, les reins, les branchies et le cœur du poisson chat de la rivière Ogun au Nigeria. $\mathrm{La}$ faible concentration du $\mathrm{Cd}$ dans les reins dans notre étude comme dans les autres plus antérieures (Farombi et al., 2007) peut être due à une faible tendance du $\mathrm{Cd}$ envers les sites actifs disponibles (azote et/ou oxygène, atomes donneurs) dans les tissus des reins pour former le Cd (II) tétraèdre (ou carré) qui est plus complexe (Kappus, 1987). Toutefois, les concentrations très élevées des métaux dans notre étude peuvent être dues à l'ampleur de la pollution due à l'usine de la SNPT.

Dans l'ensemble, les concentrations des métaux au niveau des sites pollués sont supérieures aux normes admises pour une consommation (WHO, 1992) indiquant une contamination des poissons aux métaux lourds et en tant que tel par la chaîne alimentaire, les consommateurs peuvent êtres vulnérables à la toxicité des métaux lourds toxiques.

Nos résultats ont montré une élévation significative de la peroxydation lipidique dans tous les organes des poissons des sites pollués mais surtout à Goumoukopé par rapport à la zone de référence (Gbodjomé). Cette augmentation peut être attribuée à l'accumulation du $\mathrm{Cd}$ et du $\mathrm{Pb}$ dans les organes tels que l'indiquent nos données. En effet, l'accumulation de ces métaux catalyse les ROS capables d'endommager les biomolécules comme l'ADN, les protéines et les lipides (Pandey et al., 2003 ; Farombi et al., 2007 ; Yildirim et al., 2011).

L'activité de la GST est élevée dans tous les organes sauf au niveau des branchies à Agbodrafo et goumoukopé où elle a augmenté par rapport aux échantillons contrôles. Cette élévation significative de la GST dans les organes peut être une réponse au stress oxydatif causé par la présence des métaux lourds dont l'accumulation conduirait à une production d'ions superoxydes qui induisent le superoxyde dismutase (SOD) pour transformer le radical superoxyde en $\mathrm{H}_{2} \mathrm{O}_{2}$ (Kadar et al., 2005). La GSH est reconnue comme un substrat de l'activité de la GST (Farombi, 2007). L'augmentation apparente des taux de la GSH avec ceux de la GST dans les organes de $S$. barracuda des zones polluées par rapport à ceux de Gbodjomé suppose un rôle adaptatif et protecteur de cette biomolécule contre le stress oxydatif provoqué par les métaux lourds. Nos résultats sont similaires à ceux 
d'autres travaux (Pandey et al., 2003; Farombi et al., 2007) respectivement sur le poisson Wallago attu de la rivière parripat en Inde et sur le poisson chat de la rivière Ogun au Nigeria. Les faibles taux de la GST et de la GSH dans les branchies à Agbodrafo et Goumoukopé pourraient justifier la peroxydation lipidique sensible observée. Les branchies sont plus exposées à l'eau contaminée et donc, un métal peut pénétrer dans les fines cellules épithéliales (Gul et al., 2004). Sous l'effet d'un stress oxydatif aigu, les effets toxiques des polluants peuvent accabler les défenses antioxydantes (Bebianno et al., 2004). Aussi, l'apparente diminution du système de détoxification de la glutathion (GSH) dans les branchies, premier point de contact avec les xénobiotiques environnementaux confirme que ce système est un indicateur sensitif biochimique de la pollution environnementale (Kono et al., 1982) chez le barracuda. Aussi, cette variation particulière de la GSH et de la GST montre que ces zones sont plus touchées par le rejet des déchets phosphatés. En effet, Agbodrafo et Goumoukopé sont des zones limitrophes de Kpémé (lieu de rejet des boues phosphatées). De plus, ces zones font objet des rejets des déchets phosphatés dans la mer et l'eau de mer coule vers Goumoukopé amenant ainsi ces déchets dans cette localité. Dans la zone marine d'Aného située encore plus loin à environ $9 \mathrm{Km}$, la biodiversité marine y subi des effets plus atténués mais non négligeables.

L'augmentation de l'activité de la catalase est souvent observée en présence des polluants environnementaux (Dautremepuits et al., 2004 ; Farombi et al., 2007) car le système superoxyde dismutase-catalase (SOD-CAT) représente la première ligne de défense contre le stress oxydatif (Yildirim, 2011). Cependant, dans notre étude, l'activité de la CAT a diminué dans tous les organes des poissons à Goumoukopé et a augmenté à Agbodrafo et Aného par rapport à celle de Gbodjomé (contrôle). La réduction de cette activité à Goumoukopé serait due au flux des radicaux superoxydes qui empêchent l'activité de la catalase (Stanic et al., 2005), vu la position de cette zone qui expose d'avantage les espèces marines aux polluants. Cette réduction de l'activité de la catalase a été précédemment rapportée chez les Cyprinidae vivants dans le barrage de Seyhan construit sur le lac de Turquie (Gul, 2004); chez l'Alipenser ruthenus L. de la rivière Danube en Serbie (Stanic et al., 2005) et chez le poisson chat de la rivière Ogun au Nigéria (Farombi et al., 2007). Toutefois, l'augmentation observée dans les deux autres zones montre une lutte contre le stress oxydatif produit par les métaux lourds contenus dans les déchets rejetés par l'usine de traitement des phosphates du Togo dans la mer.

\section{Conclusion}

D’une façon collective, la présente étude montre que les fortes concentrations du $\mathrm{Cd}$ et $\mathrm{du} \mathrm{Pb}$ accumulés dans le foie, les branchies, les reins et le cœur de $S$. barracuda de la côte marine togolaise au niveau d'Agbodrafo, Goumoukopé et Aného sont dues à l'activité industrielle de la SNPT qui rejette des déchets phosphatés dans la mer à ces niveaux. Ceci serait la cause de l'altération des activités des enzymes antioxydantes et d'autres biomarqueurs du stress oxydatif chez le $S$. barracuda qui peut causer un disfonctionnement biochimique chez cette espèce ainsi que chez d'autres espèces de la biodiversité marine dans cette partie de la côte togolaise. Ces résultats montrent que les biomarqueurs enzymatiques et non enzymatiques du stress oxydatif sont des indicateurs de la pollution aquatique. Aussi, est-il nécessaire d'ajouter que cette pollution, si elle touche directement Kpémé, lieu de rejet des boues, ses effets sont également très intense à Goumoukopé de part sa position par rapport à Kpémé, intense à Agbodrafo plus proche de Kpémé vers l'amont et non négligeable jusqu'à Aného à cause du drainage des déchets par la mer vers cette localité. 


\section{REFERENCES}

Abbe K. 2004. Evaluation des conséquences du rejet des déchets phosphatés dans la mer: Etude de la bioaccumulation de quelques éléments chimiques toxiques par les espèces animales marines (cas du Cadmium et du Plomb). Mémoire en GEE/ESTBA, Université de Lomé, p. 6 ; $21 ; 25 ; 29-30$.

Aebi H. 1984. Catalase in vitro. Methode in Enzymole, 105: 121-126.

Ahmad I, Hamid T, Fatima M, Chand H-S, Jain S-K, Athar M, Raisuddin S. 2000. Induction of hepatic antioxydants in freshwater catfish (Channa punctatus Bloch) is a biomarker of paper mill effluent exposure. Biochimica et Biophysica Acta, 1523: 37-48.

Aït-Aïssa S, Palluel O, Porcher J-M. 2003. Biomarqueurs précoces d'écotoxicité. Rapport final BCRD, Ministère de l'Ecologie du Développement Durable, INERIS, Paris.

Alméida J-A, Diniz Y-S, Marques S-F-G, Faine L-A, Ribas B-O, Burneiko R-C, Novelli E-L-B. 2002. The use of the oxydative stress response as biomarkers in Nile tilapia (Oreochromis niloticus) exposed to in vivo cadmium contamination. Environment International, 27: 673-679.

Asharaf W. 2005. Accumulation of heavy metals in kidney and heat tissues of Epinephelus microdon fish from the Arabian Gulf. Environ. Monit. Assess., 101: 311.

Awade M. 2010. Les impacts environnementaux et socioéconomiques de l'exploitation du gisement de phosphate de Hahotoé-Kpogamé. Mémoire de Maîtrise ès Lettre et Sciences Humaines, Option Géomorphologie et Gestion des Milieux Naturels, p. 80-82.

Bebianno M-J, Geret F, Hoarau P, Serafim MA, Coelho M-R, Gnassia-Barelli M, Romeo M. 2004. Biomarkers in Ruditapes decussates : a potential bioindicator species. Biomarkers, 9: 305330.
Bradford M. 1976. A rapid and sensitive method for the quantitation of microgram quantities of proteins utilizing the principale of protein dye - binding. Anal. Biochem., 72: 248-254.

Charissou A-M, Cossu-Leguille C, Vasseur P. 2004. Relationship between two oxidative stress biomarkers, malondialdehyde and 8-oxo-7,8-dihydro-2'-deoxyguanosine, in the freshwater bivalve Unio tumidus. Sci. Total Environment, 322: 109-122.

Chinni S, Yallapragda R. 2000. Toxicity of copper, cadmium, zinc and lead to Pnaeus indicus postlarvae: effects of individual metals. J. Environ. Biol., 21: 255-258.

Dautremepuits C, Paris-Palacios S, Betoulle S, Vernet G. 2004. Modulation in hepatic and head kidney parameters of carp (Cypinus carpio L.) induced by copper and chitosan. Comp. Biochem. Physiol. C Toxicol. Pharmacol., 137: 325-333.

Farombi E-O, Adelowo O-A, Ajimoko Y-R. 2007. Biomarkers of oxidative stress and heavy metal levels as indicators of environmental pollution in African Cat Fish (Clarias gariepinus) from Nigeria Ogun River. International Journal of Environmental Research and Public Health, 4(2): 158-165.

Gnandi K. 1998. Cadmium et autres polluants inorganiques dans les sols et les sédiments de région côtière du Togo : étude géochimique. Thèse de l'Université Friedrich Alexandre d'Erlangen Nuremberg, Republique fédérale d'Allemagne, p. 10-11; 137.

Gul S, Belge-Kurutas E, Yildiz E, Sahan A, Doran F. 2004. Pollution correlated modifications of liver antioxidant systems and histopathology of fish (Cyprinidae) living in Seyhan Dam lake, Turkey. Environ. Int., 30: 605-609.

Habig W-H, Pabst M-J, Jacoby W-B. 1974. Glutathione-s-transferases. The first enzymatic step in mercapturic acid formation. J. Biol. Chem., 249: 71307139. 
Halliwell B, Gutteridge J. 1999. Free Radicals in Biology and Medicine ( $3^{\text {rd }}$ edn). Oxford University Press: Oxford; 617-783.

Jollow D-J, Michell J-R, Zampaglione N, Gillete J-R. 1974. Bromobenzene induced liver Necrosis: protective role of GSH and evidence for 3,4-Bromobenzene oxide as the hepatotoxic metabolite. Pharmacology, 11: 151-169.

Kadar E, Costa V, Santos R-S. 2005. Distribution of micro-essential $(\mathrm{Fe}, \mathrm{Cu}$, $\mathrm{Zn})$ and toxic $(\mathrm{Hg})$ metals in tissues of two nutritionally distinct hydrothermal shrimps. Sci. Total Environ., 2005: 65-70.

Kalay M, Ay P, Canil M. 1999. Heavy metal concentration in fish tissues from the northeast Meditereansea. Bul. Environ. Contam. Toxicol., 63: 673-671.

Kappus H.1987. Oxidative stress in chimical toxicity. Arch. Toxicol., 60: 144-149.

Kargin F, Cogin H-Y. 1999. Metal interactions during accumulation and elimination of zinc and cadmium in tissues of the fish water fish, Tilapia nilonitica. Bull. Environ. Contam. Toxicol., 63: 511-519.

Kono Y, Fridovich I. 1982. Superoxide radical inhibits catalase. J. Biol. Chem., 257(1-4): 575.

Lin C-T, Lee T-L, Duan K-J, Su J-C. 2001. Purification and characterization of black porgy muscle $\mathrm{Cu} / \mathrm{Zn}$ Superoxyde dismutase. Zoological Studies, 4(2): 8490.

Livingstone D-R. 2001. Contaminantstimulated reactive oxygen species production and oxidative damage in aquatic organisms. Marine Pollution Bulletin, 42: 656-666.

Nordberg J, Arner E-S-J. 2001. Reactive oxygen species, antioxidants and the mammalian thiredoxin system. Journal of Free Radicals in Biology and Medicine, 31(11): 1287-1312.

Pandey S, Parvez S, Sayeed I, Haque R, BinHafeez B, Raisuddin S. 2003. Biomarkers of oxidative stress: a Comparative study of river Yamuna Fish Wallago attu (BI. \& Schn.). Sci. Total Environ., 309: 105-115.

Satoh K. 1978. Serum lipid peroxide in cerebrovascular disorders determined by a new colorimetric method: $\mathrm{Cl}$. Chim. A., 90: $37-43$.

Stanic B, Andric N, Zoric S, Grubor-Lajsic G, Kovacevic R. 2005. Assessing pollution in the Danube River near Novi Sad (Serbia) using several biomarkers in sterlet (Acipenser ruthenus L.). Ecotoxicol. Environ. Safety, 65(3): 395402.

Sole M, Porte C, Biosca X, Mitchelmore C-L, Chipman J-K, Livingstone D-R, Albaiges J. 1996. Effects of the "Aegean Sea" oil spill on biotransformation enzymes, oxidative stress and DNA-Adducts in digestive gland of the mussel (Mytilus edulis L.), Comparative Biochemistry and Physiology Part C. Toxicol. Pharmaco., 113: 257-265.

Tchangbedji G, Djeteli G, Kili A, Savariault M-J, Lacoutl J. 2003. Chimical and structural characterization of natural phosphate of Hahotoé (Togo). Bul. Chim., Ethiop., 17(2): 1-8.

Velkova-Jordanoska L, Kostoski G, Jordanoska B. 2008. Antioxidative enzymes in fish as biochemical indicators of aquatic pollution. Bulgarian J. Agric. Sci., 14(2): 235-237.

Viana F, Huertas R, Danulat E. 2005. Heavy metal levels in fish from coastal waters of Uruguay. Arch. Environ. Contam. Toxicol., 48: 530-537.

WHO. 1992. Inorganic Lead. Environmental Health Criteria, Vol. 165. WHO: Geneva.

Yildirim N-C, Benzer F, Danabas D. 2011. Evaluation of environmental pollution at Munzur river of Tunceli applying oxidative stress biomarkers in Cappota trutta (Heckel, 1843). J. Anm. Plant Sci. 21(1): 66-71. 